\title{
Descripción y Caracterización de las Fracturas Mandibulares Atendidas en un Servicio de Urgencia de un Hospital Público de Santiago, Chile
}

\author{
Description and Characterization of Mandibular Fractures Treated \\ in an Emergency Service of a Public Hospital in Santiago, Chile
}

Joaquín Vázquez'; Alfio Secchi'; Felipe Astorga ${ }^{2,3}$; Jorge Moreno ${ }^{3}$ \& Pablo Amorin ${ }^{3}$

VÁZQUEZ, J.; SECCHI, A.; ASTORGA, F.; MORENO, J. \& AMORIN, P. Descripción y caracterización de las fracturas mandibulares atendidas en un servicio de urgencia de un hospital público de Santiago, Chile. Int. J. Odontostomat., 15(1):278-285, 2021.

RESUMEN: Las fracturas mandibulares son parte importante del trauma maxilofacial presentando consecuencias funcionales y estéticas importantes si su manejo inicial es inadecuado o tardío. Estudios epidemiológicosde lesiones aportan en orientar esfuerzos y recursos parasuevaluación y manejo. Objetivo del estudio es caracterizar variables asociadas a pacientes con fracturas mandibulares, atendidos en la Unidad de Emergencia del Complejo Asistencial Barros Luco (CABL). Estudio retrospectivo fue realizado con los registros clínicos de pacientes atendidos entre enero 2018 y junio 2019 con diagnóstico de fractura mandibular. Se consignó sexo, edad, número de fracturas, localización anatómica, etiología, TRIAGE de entrada según ESI, presencia de TEC, medio de transporte al ingreso y comuna de procedencia. Los 57 Pacientes presentaron al menos una fractura mandibular, sexo masculino presentópredominio,edad promedio 31 años. Población entre 20 y 29 años fue más prevalente. 82 fracturas fueron consignadas siendo ángulo mandibular izquierdo la principal zona afectada $(n=15,18,3 \%) .8$ pacientes presentaron TEC, clasificados como ESI 3 y ESI 2. Demanda predominante fue de pacientes pertenecientes al SSMS (54,4\%).Los patrones etarios y localización de las fracturas encontrados coinciden con lo publicado previamente, con una disminución de brecha de casos entre hombres y mujeres, la asociación etiológica con estructuras anatómicas comprometidas y la presencia de TEC en casos de mayor severidad. Queda para analizar el porqué importante carga que presenta el CABL es de pacientes no pertenecientes a su jurisdicción $(45,6 \%)$. Incidencia y causas de fracturas mandibulares reflejan diversos patrones de comportamiento entre comunidades. Se reafirma como factor de riesgo la edad, sexo, observando una mayor prevalencia de fracturas enángulo mandibular izquierdo, asociado a patrones de comportamiento sociocultural. Sugerimos realizar correcciones metodológicas en investigaciones futuras con un proceso de estandarización de registro porlos profesionales encargados de consignar los registros clínicos.

PALABRAS CLAVE: epidemiología, fracturas mandibulares, traumatismo craneoencefálico

\section{INTRODUCCIÓN}

Las Fracturas Mandibulares son una de las lesiones traumáticas más frecuentes del territorio maxilofacial, debido en parte a la extensión del área que conforma en este territorio, asi como también a su anatomía que aporta función de movilidad, lo cual frente a traumatismos favorece la pérdida de estabili- dad y con ello resistencia. Bajo este escenario la estructura mandibular permanece en exposición constante ante injurias, siendo sus principales causas descritas las agresiones físicas, accidentes automovilísticos, caídas, accidentes deportivos, entre otras (Czerwinski et al., 2008).

\footnotetext{
${ }^{1}$ Cirujano Dentista, Pasante Servicio Cirugía Maxilofacial Complejo Asistencial Barros Luco, Santiago, Chile.

${ }^{2}$ Cirujano Maxilofacial, Unidad de Urgencia Dental Complejo Asistencial Barros Luco, Santiago, Chile.

${ }^{3}$ Cirujano Dentista, Unidad de Urgencia Dental Complejo Asistencial Barros Luco, Santiago, Chile.
} 
Su etiología e incidencia varían entre reportes de acuerdo con la localización geográfica, estatus socioeconómico, leyes de tránsito, cultura local y época en la cual han sido estudiadas. Los accidentes automovilísticos han sido reportados como principal causa en países en vías de desarrollo, mientras que en países desarrollados es la violencia interpersonal (Simsek et al., 2007).

Dentro de sus principales secuelas, la infección posee una alta prevalencia, dado la alta carga bacteriana oral comunicada hacia espacios anatomicos aislados; de igual manera la disminución de la eficiencia masticatoria, el compromiso estético, dolor crónico y trastornos temporomandibulares han sido descritos. La probabilidad de presentación de estas secuelas aumenta conforme su manejo inicial es más tardío (Fischer et al., 2001).

El Traumatismo Encéfalo Craneano (TEC) está generalmente asociado al Trauma Maxilofacial, siendo definido como una injuria no degenerativa a nivel cerebral, producto de una una fuerza externa, la cual puede llevar a impedimentos cognitivos, físicos y sicológicos con una disminución o alteración de la conciencia, efectos que pueden ser temporales o permanentes, exponiendo al paciente a escenarios de riesgo de alta morbilidad y mortalidad en casos severos; el análisis de respuesta ocular, verbal y motora de la escala de Glasgow permiten determinar compromiso, determinando la necesidad de un manejo rápido (Dawodu, 2019). Sus presentaciones más comunes son la contusión cerebral, daño axonal difuso, hematoma epidural, hematoma subdural y hemorragia subaracnoidea (Kreipke \& Rafols, 2012).

Producto de su relación anatómica, la presencia de lesiones traumáticas faciales como lo son las fracturas mandibulares nos debe orientar a buscar la detección de TEC en la evaluación Pre-hospitalaria, al momento del ingreso al servicio de urgencia, evaluaciones posteriores y controles Post-operatorios.

El Complejo Asistencial Barros Luco (CABL), Hospital de alta complejidad del sector sur de Santiago de Chile es el centro de referencia de atención del Servicio de Salud Metropolitano Sur (SSMS), con una población inscrita cercana a los 2 millones de personas, razón por la cual se maneja gran parte del trauma maxilofacial que se presenta en la ciudad. Estudios a nivel nacional recientes, han descrito de buena manera la epidemiología de las fracturas maxilofaciales atendidas en hospitales del país, contabilizando las cirugías realizadas en los servicios de cirugía maxilofacial, mas no describiendo la realidad de las atenciones dadas en las Unidades de Urgencia (Perez et al., 2015; Faille Horwood \& Badillo Coloma, 2018; Cuellar et al., 2019). Resulta importante analizar la realidad propia de las Unidades de Urgencia de los centros hospitalarios, considerando que parte de los pacientes no son derivados a los Centros de Diagnóstico y Tratamiento (CDT), siendo controlados en las mismas Unidades de Urgencia.

El objetivo de este estudio es hacer una descripción de los patrones de fracturas mandibulares que se reciben en la Unidad de Emergencia de este centro hospitalario, analizando su distribución por edad, sexo, número de fracturas, su localización anatómica, agente etiológico, TRIAGE de entrada, la presencia de TEC, medio de llegada al complejo asistencial y Servicio de Salud en el cual se encuentra inscrito el paciente.

\section{MATERIAL Y MÉTODO}

Se realizó un estudio retrospectivo observacional con los ingresos de pacientes a la Unidad de Urgencia del CABL entre enero 2018 y junio 2019 (18 meses). Para la recolección de antecedentes fueron revisados los Datos de Atención de Urgencia (DAU), documento de registro clínico electrónico de las atenciones en la unidad de urgencia. Se trabajó con todo paciente que presentara como diagnóstico una o más fracturas mandibulares, siendo excluidos todos los pacientes cuyo registro clínico se encontrara incompleto, o que consultaron como control posterior a una atención inicial de las lesiones.

Los datos extraídos de los DAU fueron sexo, edad, número de fracturas, localización anatómica (condilar, subcondilar, rama, ángulo, sínfisis y parasínfisis), TRIAGE de entrada por clasificación Emergency Severity Index (ESI), agente etiológico (agresión física, accidentes automovilísticos, accidentes domésticos, accidentes escolares), presencia de TEC, medio de llegada al servicio (vehículo particular, ambulancia, a pié, transporte público, patrulla policial), y comuna de pertenencia a red de salud del paciente.

La información fue transferida a una planilla Microsoft Excel. Para variables continuas se calculó media y desviación estándar, mientras que variables categóricas fueron expresadas en su total y como porcentaje. 


\section{RESULTADOS}

Entre enero 2018 y junio de 2019 un total de 57 pacientes ingresó con al menos una fractura mandibular, siendo 82 fracturas registradas entre todos los registros.

Edad y distribución por sexo. La Edad promedio de los pacientes fue 31 años con 12 años de desviación estándar. El paciente más joven y de mayor edad fueron 16 y 67 años respectivamente. Pacientes entre los 20 a 29 años fueron el mayor porcentaje de consultas (22 pacientes, 38,6 \%) con un ratio entre población masculina y femenina de 2,67:1. Los siguen los grupos entre 30 a 39 años (14 pacientes, $24,6 \%$ ), y menores de 20 años (9 pacientes, 15,8\%). El ratio entre población masculina y femenina entre toda la población fue de 4,18:1 (Tabla I).

Número de Fracturas por paciente diferenciado por sexo. En los 57 pacientes estudiados, la fractura mandibular única fue la más frecuente tanto en población masculina como femenina (37 pacientes, $64,9 \%$, ratio $3,6: 1$ ), seguido de la fractura doble (16 pacientes, $28,1 \%$, ratio $4,3: 1)$. Baja frecuencia de fracturas triples y cuádruples fueron encontradas, siendo solo 4 casos entre ambos grupos unicamente con población masculina afectada (Tabla II).

Sitio de Fracturas. Un total de 82 fracturas fueron registradas en 57 pacientes, sufriendo 20 de estos fracturas múltiples mandibulares (35,1\%). La localización anatómica más afectada fue el ángulo mandibular siendo el lado izquierdo el más afectado, en comparación al derecho (29 fracturas, $25 \%$, ratio 1,07:1), seguido de sínfisis/parasínfisis con igual distribución bilateral (16 fracturas, $19,5 \%$, ratio $1: 1$ ), cóndilo y cuerpo mandibular presentaron igual prevalencia (14 fracturas, $17,1 \%$ ), dejando como zona menos afectada la rama mandibular (9 fracturas, $11 \%$ ) (Tabla III).

Mecanismo de Trauma, TRIAGE de entrada y Presencia de TEC. 21 ingresos al servicio fueron consignados como accidentes domésticos siendo este el principal mecanismo de trauma reportado, seguido de agresiones con 20 casos y accidentes de tránsito con 15. Solo 1 ingreso fue registrado como accidente escolar.

Tabla I. Pacientes atendidos por Fractura Mandibular según sexo y edad.

\begin{tabular}{cccccc}
\hline Edad & Masculino & Femenino & Total & Porcentaje & Ratio \\
\hline$<20$ & 8 & 1 & 9 & 15.8 & $8: 1$ \\
$20-29$ & 16 & 6 & 22 & 38.6 & $2.67: 1$ \\
$30-39$ & 12 & 2 & 14 & 24.6 & $6: 1$ \\
$40-49$ & 8 & 0 & 8 & 14.0 & $8: 1$ \\
$50-59$ & 1 & 1 & 2 & 3.5 & $1: 1$ \\
$>60$ & 1 & 1 & 2 & 3.5 & $1: 1$ \\
Total & 46 & 11 & 57 & 100 & $4.18: 1$ \\
\hline
\end{tabular}

Tabla II. Número de Fracturas Mandibulares por paciente según sexo.

\begin{tabular}{lccccc}
\hline \multicolumn{1}{c}{ Número de sitios de fractura } & Masculino & Femenino & Total & Porcentaje & Ratio \\
\hline Fractura única & 29 & 8 & 37 & 64.9 & $3.6: 1$ \\
Fractura doble & 13 & 3 & 16 & 28.1 & $4.3: 1$ \\
Fractura triple & 3 & 0 & 3 & 5.3 & - \\
Fractura cuadruple & 1 & 0 & 1 & 1.8 & - \\
Total & 46 & 11 & 57 & 100 & $4.18: 1$ \\
\hline
\end{tabular}

Tabla III. Posición anatómica de fracturas y lado más afectado.

\begin{tabular}{lccccc}
\hline \multicolumn{1}{c}{ Posición } & Izquierda & Derecha & Total & Porcentaje & Ratio \\
\hline Cóndilo mandibular & 5 & 9 & 14 & 17.1 & $1: 1.8$ \\
Rama mandibular & 4 & 5 & 9 & 11 & $1: 1.25$ \\
Ángulo mandibular & 15 & 14 & 29 & 25.4 & $1.07: 1$ \\
Cuerpo mandibular & 7 & 7 & 14 & 17.1 & $1: 1$ \\
Sínfisis / Parasínfisis & 8 & 8 & 16 & 19.5 & $1: 1$ \\
Total & 39 & 43 & 82 & 100 & $1: 1.1$ \\
\hline
\end{tabular}


Según TRIAGE de entrada valorado bajo escala ESI, la mayor parte de los pacientes presentaban bajo riesgo de deterioro próximo (ESI 4, 37 pacientes, 64,9 $\%$ ), seguido de pacientes estables (ESI 3, 18 pacientes, $31,6 \%$ y un bajo porcentaje de pacientes con alto riesgo de deterioro próximo (ESI 2, 2 pacientes, 3,5 \%). No se registraron ingresos ESI 1 ni ESI 5 (Tabla IV).

En 6 pacientes ESI 3 y los 2 pacientes ESI 2 fue consignado TEC posterior a la evaluación completa del equipo de medicina de urgencia, representando un 14 $\%$ de los casos.

Distribución por Servicio de Salud y medio de ingreso a la Unidad de Emergencia. 31 pacientes ins- critos en el SSMS fueron registrados $(54,4 \%$ de las consultas), mientras que 26 pacientes pertenecían a otros Servicios de Salud (45,6 \% de las consultas). De las comunas pertenecientes al SSMS, la que más pacientes aportó al registro fue San Bernardo (10 pacientes, 17,5\%), seguida de El Bosque (7 pacientes, $12,3 \%$ ), Pedro Aguirre Cerda y San Miguel (4 pacientes, $7 \%$ ).

El medio de ingreso a la unidad de emergencia más ocupado fue el transporte particular (Vehículo motorizado, 25 pacientes), seguido del transporte público o privado (19 pacientes) y ambulancia en tercer lugar (8 pacientes) (Tabla V).

Tabla IV. Incidencia de Trauma y categorización ESI al ingreso al servicio.

\begin{tabular}{ccccccc}
\hline ESI & Agresión & $\begin{array}{c}\text { Accidente de } \\
\text { Tránsito }\end{array}$ & $\begin{array}{c}\text { Accidente } \\
\text { Doméstico }\end{array}$ & $\begin{array}{c}\text { Accidente } \\
\text { escolar }\end{array}$ & Total & Porcentaje \\
\hline 1 & 0 & 0 & 0 & 0 & 0 & 0 \\
2 & 1 & 0 & 1 & 0 & 2 & 3.5 \\
3 & 3 & 9 & 6 & 0 & 18 & 31.6 \\
4 & 16 & 6 & 14 & 1 & 37 & 64.9 \\
5 & 0 & 0 & 0 & 0 & 0 & 0 \\
Total & 20 & 15 & 21 & 1 & 57 & 100 \\
\hline
\end{tabular}

Tabla V. Distribución por servicio de salud, comuna y medio de llegada.

\begin{tabular}{|c|c|c|c|c|c|c|c|c|}
\hline $\begin{array}{l}\text { Servicio de } \\
\text { salud de } \\
\text { pertinencia }\end{array}$ & Comunas & Ambulancia & $\begin{array}{l}\text { Vehículo } \\
\text { motorizado }\end{array}$ & A Pie & $\begin{array}{l}\text { Patrulla } \\
\text { policial o } \\
\text { PDI }\end{array}$ & $\begin{array}{l}\text { Transporte } \\
\text { público o } \\
\text { privado }\end{array}$ & Total & Porcentaje \\
\hline & San Bernardo & 1 & 5 & 1 & 0 & 3 & 10 & 17.5 \\
\hline & $\begin{array}{l}\text { Pedro Aguirre } \\
\text { Cerda }\end{array}$ & 2 & 1 & 0 & 0 & 1 & 4 & 7 \\
\hline & El Bosque & 1 & 2 & 1 & 0 & 3 & 7 & 12.3 \\
\hline \multirow[t]{5}{*}{ SSMS } & San Miguel & 1 & 1 & 0 & 1 & 1 & 4 & 7 \\
\hline & San Joaquín & 0 & 2 & 0 & 0 & 1 & 3 & 5.3 \\
\hline & Calera de Tango & 0 & 0 & 0 & 0 & 1 & 1 & 1.8 \\
\hline & La Cisterna & 0 & 0 & 1 & 0 & 0 & 1 & 1.8 \\
\hline & Buin & 0 & 0 & 0 & 0 & 1 & 1 & 1.8 \\
\hline \multirow[t]{2}{*}{ Otro } & & 3 & 14 & 1 & 0 & 8 & 26 & 45.6 \\
\hline & Total & 8 & 25 & 4 & 1 & 19 & 57 & 100 \\
\hline
\end{tabular}

\section{DISCUSIÓN}

Un total de 57 pacientes con diagnóstico de fractura mandibular atendidos entre enero 2018 y junio 2019 en el Servicio dental de la Unidad de Emergencia del $\mathrm{CABL}$ fueron incluidos en la presente revisión. A pesar de que los hallazgos tienden a ser similares con lo reportado en la literatura, algunos datos nos pueden dar luces de únicas influencias geográficas, culturales y socioeconómicas de cada región estudiada.
Pacientes entre 20 y 29 años ( $n=22,38,6 \%)$ fueron el principal grupo afectado. A pesar del tamaño muestral obtenido en este trabajo similares resultados han sido descritos en estudios extranjeros, con principal prevalencia en los grupos entre 21 y 30 años. Yu et al. (2011) evaluaron la frecuencia de fracturas mandibulares y de sus patrones de fractura reportando similar prevalencia en los grupos de 21 a 30 años 
$(n=62,31 \%)$. Importante mencionar que el grupo entre 30 y 39 años es casi un cuarto de los casos $(n=14$, $24,6 \%$ ), lo que nos recalca la importancia de la educación continua respecto a conductas de riesgo en las que pueden estar incurriendo a lo largo de la vida de una persona, reforzando esto a grupos jóvenes y adultos (Yu et al.).

En concordancia con la literatura tradicional se aprecia predominancia masculina en los casos por sobre la femenina (hombres $=46$, mujeres $=11$ ). El ratio obtenido entre los sexos $(4,18: 1)$ presenta una menor diferencia de casos entre estos grupos. Lin et al. (2017) presentaron la relación más pareja presentada hasta el momento entre población masculina y femenina $(1,8: 1)$, situación similar a lo encontrado por Obimakinde et al. (2017)my Chalya et al. (2011), donde mostraban una disminución de la discrepancia de casos entre hombres y mujeres en los últimos años. Esta información junto a nuestros datos nos expone la premisa de un aumento de prevalencia de atenciones en mujeres en los últimos 10 años. Esto se puede asociar a la mayor incorporación de la población femenina al mundo laboral, elemento que al exponer a mujeres a mayor interacción con el medio como el transporte, asaltos y/o agresiones ha hecho aumentar su incidencia, especialmente en países en vías de desarrollo, sin descartar el aumento de agresión por violencia intrafamilar o de género, datos que en este estudio no podemos diferenciar (Chalya et al.; Lin et al.; Obimakinde et al.) (Tabla VI).
Las localizaciones anatómicas más comprometidas en orden decreciente fueron el ángulo mandibular izquierdo ( $n=15,18,3 \%$ ), ángulo mandibular derecho $(n=14,17,1 \%)$, sínfisis y parasínfisis mandibular $(n=16$, $19,5 \%$ ), cóndilo y cuerpo mandibular con igual distribución ( $\mathrm{n}=14$ cada sitio, $17,1 \%$ ), y rama mandibular $(n=9,10,9 \%)$. La distribución que presentamos es similar a lo reportado en población estadounidense teniendo al ángulo mandibular como principal sitio afectado, argumentado que esta área posee una menor resistencia asociada a factores como dientes incluidos, menor grosor del margen basilar y el cambio de dirección estructural de la macro y microarquitectura ósea (Ogundare et al., 2003); en lo correspondiente a la alta frecuencia de afección del lado izquierdo, se puede asociar con la violencia interpersonal en donde la mayor población posee habilidades de coordinación diestra. En población africana y asiática se presenta la sínfisis y cóndilo como principales sitios afectados, los autores discuten esta distribución particular como consecuencia del alto uso de medios de transporte individuales como motocicletas y el no uso de casco de seguridad (Lin et al.; Krishnaraj \& Chinnasamy, 2007), mientras que nuestra realidad asociada tradicionalmente a trauma maxilofacial local se asocia más cuadros de violencia interpersonal (Tabla VII).

Los accidentes domésticos fueron reportados en nuestra revisión como principal causa del trauma $(n=21,36,8 \%)$, seguido de cerca por agresiones $(n=20$, $35,1 \%)$ y de accidentes de tránsito ( $n=15,26,3 \%)$.

Tabla VI. Distribución por sexo entre diversas poblaciones.

\begin{tabular}{cccccccc}
\hline Estudio & $\begin{array}{c}\text { Santiago, } \\
\text { Chile (2020) }\end{array}$ & $\begin{array}{c}\text { Taichung, } \\
\text { Taiwan } \\
(2017)\end{array}$ & $\begin{array}{c}\text { Tasmania, } \\
\text { Australia } \\
(2002)\end{array}$ & $\begin{array}{c}\text { Friburgo, } \\
\text { Alemania } \\
(2009)\end{array}$ & $\begin{array}{c}\text { Washington } \\
\text { DC, USA } \\
(2003)\end{array}$ & $\begin{array}{c}\text { Ekiti Odo, } \\
\text { Nigeria } \\
(2017)\end{array}$ & $\begin{array}{c}\text { Mwanza, } \\
\text { Tanzania } \\
(2011)\end{array}$ \\
\hline Hombres (\%) & $46(80,1 \%)$ & $127(64 \%)$ & $205(82 \%)$ & $329(74 \%)$ & $464(86 \%)$ & $180(77,3 \%)$ & $112(72,2 \%)$ \\
Mujeres (\%) & $11(19,9 \%)$ & $71(36 \%)$ & $46(18 \%)$ & $115(26 \%)$ & $76(14 \%)$ & $53(22,7 \%)$ & $42(27,3 \%)$ \\
ratio H:M & $4,18: 1$ & $1.8: 1$ & $4.5: 1$ & $2.9: 1$ & $6.1: 1$ & $3.4: 1$ & $2.7: 1$ \\
\hline
\end{tabular}

Tabla VII. Localización de sitios de fractura mandibular de acuerdo con el número reportado por país.

\begin{tabular}{cccccccc}
\hline \multirow{2}{*}{ Estudio } & $\begin{array}{c}\text { Santiago, } \\
\text { Chile }(2020)\end{array}$ & $\begin{array}{c}\text { Taichung, } \\
\text { Taiwan } \\
(2017)\end{array}$ & $\begin{array}{c}\text { Tasmania, } \\
\text { Australia } \\
(2002)\end{array}$ & $\begin{array}{c}\text { Friburgo, } \\
\text { Alemania } \\
(2009)\end{array}$ & $\begin{array}{c}\text { Washington } \\
\text { DC, USA } \\
(2003)\end{array}$ & $\begin{array}{c}\text { Chennai, } \\
\text { India } \\
(2007)\end{array}$ & $\begin{array}{c}\text { Montreal, } \\
\text { Canada } \\
(2008)\end{array}$ \\
\hline \multirow{6}{*}{$\begin{array}{c}\text { Ángulo } \\
\text { Localización }\end{array}$} & Sínfisis & Ángulo & Cóndilo & Ángulo & Sínfisis & Sínfisis \\
& $(25 \%)$ & $(39 \%)$ & $(32 \%)$ & $(42 \%)$ & $(36 \%)$ & $(44 \%)$ & $(29 \%)$ \\
& Sínfisis & Cóndilo & Cóndilo & Sínfisis & Cuerpo & Cóndilo & Cóndilo \\
& $(19.5 \%)$ & $(26 \%)$ & $(28 \%)$ & $(21 \%)$ & $(21 \%)$ & $(23 \%)$ & $(25 \%)$ \\
& Cuerpo & Ángulo & Sínfisis & Ángulo & Sínfisis & Alveolar & Ángulo \\
& $(17.1 \%)$ & $(14 \%)$ & $(19 \%)$ & $(20 \%)$ & $(18 \%)$ & $(12 \%)$ & $(19 \%)$ \\
& Cóndilo & Cuepro & Cuerpo & Cuerpo & Cóndilo & Cuerpo & Cuepro \\
& $(17.1 \%)$ & $(14 \%)$ & $(18 \%)$ & $(15 \%)$ & $(17 \%)$ & $(15 \%)$ & $(14 \%)$ \\
& Rama $(11 \%)$ & Rama $(7 \%)$ & Rama $(2 \%)$ & Rama $(2 \%)$ & Rama $(2 \%)$ & Rama $(9 \%)$ & Rama $(5 \%)$ \\
& & & & & & &
\end{tabular}


Estos hallazgos difieren a lo encontrado en el resto de los estudios donde agresiones o asaltos, y accidentes automovilísticos son los principales agentes etiológicos del trauma mandibular (Lin et al.; Simsek et al.; Czerwinski et al.; Krishnaraj \& Chinnasamy). Considerando la baja diferencia causal entre nuestros hallazgos (36,8 \% vs $35,1 \%$ vs $26,3 \%$ ), podemos atribuir parte del resultado al tamaño muestral obtenido menor al resto de los estudios, diferencias conductuales entre poblaciones, a las definiciones operacionales usadas para categorizar el ingreso de los pacientes y finalmente al miembro del personal de salud que ingresa tal variable al sistema de registro del DAU, asi como también la omisión por parte del agredido en su descripción (Tabla VIII).

La presencia de fracturas mandibulares nos habla sobre la alta energía ocurrida en el momento del trauma, razón por la cual debemos siempre considerar la presencia de otras lesiones dentro del examen físico, especialmente en el territorio maxilofacial. De la información obtenida para el reporte se consignó en 8 casos la presencia de TEC, siendo un $14 \%$ de la muestra total estando sólo en pacientes con ESI de mayor severidad (ESI II (100 \%) y ESI III (33,3\%)). Estudios americanos similares al nuestro reportan prevalencias similares de TEC asociado fracturas mandibulares entre un $10,5 \%$ a un $19,7 \%$ de los casos (Brasileiro \& Passeri, 2006; Allareddy et al., 2011); valores que suben si se consideran fracturas de macizo facial completo llegando la asociación con TEC a rangos de un
$67 \%$ a $71 \%$ en caso de encontrarse fracturas de órbita o patrones de fracturas extendidas Le Fort II y Le Fort III (Grant et al., 2012; Smith et al., 2012).

A pesar de ser menor la probabilidad de encontrarse TEC en presencia de fracturas mandibulares aisladas si se comparan con el resto del macizo facial, es importante recalcar la importancia de una examinación física completa y un correcto recorrido de los exámenes complementarios imagenológicos usados en el contexto de urgencia en más de una ocasión, considerando el alto porcentaje de signos no percatados en un primer momento.

El CABL al ser un centro de alta complejidad recibe gran parte de la demanda asistencial del sector sur de Santiago. Aun así, solo un $54,4 \%$ de los pacientes ingresados viven en comunas pertenecientes al SSMS, teniendo a otro $45,6 \%$ proveniente de otros sectores. Este mismo dato se asocia con la alta llegada de pacientes al CABL por sus propios medios, tanto vehículos motorizados $(n=25,48,1 \%)$ como en transporte público o privado ( $n=19,36,5 \%)$. Queda como interrogante a investigar el conocimiento de los mismos usuarios del sistema de salud sobre los servicios de salud, su pertenencia y conocimiento sobre sus centros de derivación y atención de urgencia.

El entendimiento de la epidemiología de este tipo de fracturas nos puede guiar en esfuerzos preventivos para reducir su incidencia y secuelas consecuentes.

Tabla VIII. Etiología de las fracturas mandibulares de acuerdo con su frecuencia reportada por país.

\begin{tabular}{cccccccc}
\hline Estudio & $\begin{array}{c}\text { Santiago, } \\
\text { Chile (2020) }\end{array}$ & $\begin{array}{c}\text { Taichung, } \\
\text { Taiwan } \\
(2017)\end{array}$ & $\begin{array}{c}\text { Tasmania, } \\
\text { Australia } \\
(2002)\end{array}$ & $\begin{array}{c}\text { Friburgo, } \\
\text { Alemania } \\
(2009)\end{array}$ & $\begin{array}{c}\text { Washington } \\
\text { DC, USA } \\
(2003)\end{array}$ & $\begin{array}{c}\text { Chennai, India } \\
(2007)\end{array}$ & $\begin{array}{c}\text { Montreal, } \\
\text { Canada } \\
(2008)\end{array}$ \\
\hline & A.D. & A.T. & Agresión & A.T. & Agresión & A.T. & Agresión \\
& $(36,8 \%)$ & $(82 \%)$ & $(55 \%)$ & $(32 \%)$ & $(79 \%)$ & $(87 \%)$ & $(41 \%)$ \\
Etiología (\%) & Agresión & A.D. & A.T. & Agresión & A.T. & Agresión & A.T. \\
& $(35 \%)$ & $(10 \%)$ & $(18 \%)$ & $(28 \%)$ & $(6 \%)$ & $(5 \%)$ & $(26 \%)$ \\
& A.T. & Agresión & T.D. & A.D. & H.B. & Otros & A.D. \\
& $(26,3 \%)$ & $(6 \%)$ & $(17 \%)$ & $(26 \%)$ & $(6 \%)$ & $(8 \%)$ & $(18 \%)$ \\
& A.E. & Otros & A.D. & T.D. & Otros & & T.D. \\
& $(0,3 \%)$ & $(2 \%)$ & $(5 \%)$ & $(10 \%)$ & $(9 \%)$ & & $(10 \%)$ \\
\hline
\end{tabular}

\section{CONCLUSIONES}

Nuestro estudio mantiene la tendencia global de la evidencia con el aumento de incidencia en población femenina, siendo altamente variable entre las comunidades estudiadas, mostrándose de igual manera características comunes como el alto porcentaje con causal agresiones y accidentes de tránsito, más 
allá de los hallazgos particulares de este estudio. También se sostiene un aumento en la presencia de TEC asociado a la severidad al ingreso al Servicio de Urgencia, factor que sigue siendo relevante para su pronta consigna y manejo.

El CABL presenta como gran parte de su carga asistencial la atención de población no perteneciente a su jurisdicción, levantando alarmas respecto a la capacidad resolutiva o de cobertura del resto de los servicios y del propio conocimiento de la población usuaria del Sistema de Salud y de sus Centros de Atención.

Como limitantes propias del estudio contamos con las diferencias de forma al registrar la información clínica entre los profesionales de la unidad en los DAU, lo que llevó a la exclusión de casos que presentaban fractura mandibular producto de un relato incompleto de los cuadros por no incluir la totalidad de los datos, lo que podría hacer variar los resultados obtenidos, especialmente en casos de cercanía porcentual. Estas diferencias podrían ser corregidas cambiando la metodología a un carácter prospectivo contando con un proceso de estandarización de registro entre los profesionales, lo cual sugerimos realizaren investigaciones futuras.

Resulta necesario el realizar estudios epidemiológicos que busquen comprender la realidad local de los diversos Servicios de Salud, para así mejorar la distribución y entrega de recursos en cada uno, entendiendo la realidad particular en la cual se enmarca el Trauma Mandibular y Maxilofacial en general.

VÁZQUEZ, J.; SECCHI, A.; ASTORGA, F.; MORENO, J. \& AMORIN, P. Description and characterization of mandibular fractures treated in an emergency service of a public hospital in Santiago, Chile. Int. J. Odontostomat., 15(1):278-285, 2021.

ABSTRACT: Mandible fractures are an important part of maxillofacial trauma, presenting significant and management. The objective of the study is to characterize variables associated with patientswith mandibular fractures, treated in the Emergency Unit of the Barros Luco Assistance Complex (CABL). A retrospective study was performed with the clinical records of patients seen between January 2018 and June 2019 with a diagnosis of mandibular fracture. Sex, age, number of fractures, anatomical location, etiology, input TRIAGE according to ESI, presence of ECT, means of transport at admission and municipality of origin were recorded; 57 patients presented at least one mandibular frac- ture, male sex was predominant, with an average age of 31 years. 20 and 29 yearswas more prevalent. 82 fractures were reported, with the left mandibular angle being the main affected area $(n=15,18.3 \%) .8$ patients presented ECT, classified as ESI 3 and ESI 2. The predominant demand was from patients belonging to the SSMS (54.4\%). The agepatterns and location of the fractures found coincide with that previously published, with a decrease in the gap in cases between men and women, the etiological association with compromised anatomical structures and the presence of ECT in cases of greater severity. It remains to analyze why the important burden presented by the CABL is of patients not belonging to its jurisdiction (45.6\%). Incidence and causes of mandibular fractures reflect diverse behavior patterns between communities. Age and sex are reaffirmed as a risk factor, observing a higher prevalence of left mandibular angle fractures, associated with sociocultural behavior patterns. We suggest making methodological corrections in future research with a registration standardization process by professionals responsible for recording clinical records.

KEY WORDS: epidemiology, mandible fractures, traumatic brain injury

\section{REFERENCIAS BIBLIOGRÁFICAS}

Allareddy, V.; Allareddy, V. \& Nalliah, R. P. Epidemiology of facial fracture injuries. J. Oral Maxillofac. Surg., 69(10):2613-8, 2011.

Brasileiro, B. F. \& Passeri, L. A. Epidemiological analysis of maxillofacial fractures in Brazil: a 5-year prospective study. Oral Surg. Oral Med. Oral Pathol. Oral Radiol. Endod., 102(1):28-34, 2006.

Chalya, P. L.; Mchembe, M.; Mabula, J. B.; Kanumba, E. S. \& Gilyoma, J. M. Etiological spectrum, injury characteristics and treatment outcome of maxillofacial injuries in a Tanzanian teaching hospital. J. Trauma Manag. Outcomes, 5:7, 2011.

Cuellar, G. J.; Prats, P. M. C.; Reyes, C. D. \& Sanhueza, O. V. Epidemiología del trauma maxilofacial, tratado quirúrgicamente en el Hospital de Urgencia Asistencia Pública: 3 años de revisión. Rev. Cir., 71(6):530-6, 2019.

Czerwinski, M.; Parker, W. L.; Chehade, A. \& Williams, H. B. Identification of mandibular fracture epidemiology in Canada: Enhancing injury prevention and patient evaluation. Can. J. Plast. Surg., 16(1):36-40, 2008

Dawodu, S. T. Traumatic Brain Injury (TBI) - Definition, Epidemiology, Pathophysiology. Medscape, 2019. Disponible en: https:// emedicine.medscape.com/article/326510-overview

Faille Horwood, A. \& Badillo Coloma, O. Caracterización de los casos de fracturas maxilofaciales operados en el Hospital Carlos van Buren, Chile, entre los años 2010-2014. Rev. Esp. Cir. Oral Maxilofac., 40(4):169-75, 2018.

Fischer, K.; Zhang, F.; Angel, M. F. \& Lineaweaver, W. C. Injuries associated with mandible fractures sustained in motor vehicle collisions. Plast. Reconstr. Surg., 108(2):328-31, 2001.

Grant, A. L.; Ranger, A.; Young, G. B. \& Yazdani, A. Incidence of major and minor brain injuries in facial fractures. J. Craniofac. Surg., 23(5):1324-8, 2012.

Kreipke, C. W. \& Rafols, J. A. Cerebral Blood Flow, Metabolism, and Head Trauma. The Pathotrajectory of Traumatic Brain Injury. New York, Springer, 2012. pp.170. 
VÁZQUEZ, J.; SECCHI, A.; ASTORGA, F.; MORENO, J. \& AMORIN, P. Descripción y caracterización de las fracturas mandibulares atendidas en un servicio de urgencia de un hospital público de Santiago, Chile. Int. J. Odontostomat., 15(1):278-285, 2021.

Krishnaraj, S. \& Chinnasamy, R. A 4-year retrospective study of mandibular fractures in a South Indian city. J. Craniofac. Surg., 18(4):776-80, 2007.

Lin, F. Y.; Wu, C. I. \& Cheng, H. T. Mandibular fracture patterns at a medical center in central Taiwan: a 3-year epidemiological review. Medicine (Baltimore), 96(51):e9333, 2017.

Obimakinde, O. S.; Ogundipe, K. O.; Rabiu, T. B. \& Okoje, V. N. Maxillofacial fractures in a budding teaching hospital: a study of pattern of presentation and care. Pan. Afr. Med. J., 26:218, 2017.

Ogundare, B. O.; Bonnick, A. \& Bayley, N. Pattern of mandibular fractures in an urban major trauma center. J. Oral Maxillofac. Surg., 61(6):713-8, 2003.

Perez, G. H.; Donoso, H. T.; Mardones, M. M. \& Bravo, A. R. Epidemiology of maxillofacial surgical treatments in a public hospital in Chile: a 5-year retrospective study. Int. J. Odontostomat., 9(1):37-41, 2015.

Simsek, S.; Simsek, B.; Abubaker, A. O. \& Laskin, D. M. A comparative study of mandibular fractures in the United States and Turkey. Int. J. Oral Maxillofac. Surg., 36(5):395-7, 2007.

Smith, H.; Peek-Asa, C.; Nesheim, D.; Nish, A.; Normandin, P. \& Sahr, S. Etiology, diagnosis, and characteristics of facial fracture at a midwestern level I trauma center. J. Trauma Nurs., 19(1):57-65, 2012.

Yu, Z.; Hsia, Y. \& Hsies, M. Analisys of facial bone fractures in T.S.G.H. Chin. J. Oral Maxillofac. Surg., 12:1-7, 2011.
Dirección para correspondencia:

Joaquín A. Vázquez D.

Servicio Cirugía Maxilofacial

Complejo Asistencial Barros Luco.

Santiago

CHILE

E-mail: joaquin.vazquez.d@gmail.com 